\title{
PRESUPUESTO BASADO EN RESULTADOS, (PBR) HERRAMIENTA QUE CONTRIBUYE A LA TRASPARENCIA Y EFICIENCIA HACENDARIA EN MÉXICO
}

\author{
Francisco Javier Figueroa Montaño ${ }^{1}$ \\ Lizeth Gutiérrez Molina ${ }^{2}$ \\ Jesús Guadalupe Vazquez González ${ }^{1}$ \\ Francisco Antonio Medina Ortiz ${ }^{1}$ \\ Alejandro Córdova Yánez ${ }^{1}$
}

\author{
Sumario: I.Introducción y Revisión de Literatura. II. Materiales y \\ Métodos. III. Resultados y Discusión. IV. Conclusiones
}

\section{Resumen}

La finalidad del presente trabajo consiste en hacer un esbozo de la situación actual que priva en los tres niveles de gobierno, en relación al uso del PBR, además de determinar cuál ha sido el avance en la implementación de los Presupuestos Basados en Resultado, y en su caso, los montos presupuestales que han sido objetos de MIR a nivel dependencias o secretarías. En cuanto a la región serrana de Sonora, se buscó determinar si existe conocimiento y uso del PBR, lo que significaría que los ayuntamientos están midiendo la efectividad en la aplicación del gasto público.

El presente trabajo se realizó basándose en diferentes técnicas y estrategias para la recolección de datos, en el caso de los niveles federal y Estatal, se utilizó la investigación documental. Para efecto del ámbito local, la técnica predominante fue la referida como encuesta, la cual fue aplicada

\footnotetext{
${ }^{1}$ Docentes Investigadores de la Universidad de la Sierra. División de Ciencias Económico Administrativas. Departamento de Administración y Evaluación de Proyectos. Carretera Moctezuma-Cumpas km 2.5. Moctezuma, Sonora, México. C.P.84560. Tel. (634)3429600. Email: jvazquez@unisierra.edu.mx

${ }^{2}$ Docente Investigadora del Instituto Sonorense de Administración Publica, Calle Comonfort 70 y Tehuantepec, Col. Centenario, Hermosillo, Sonora, México 83260, Teléfono +52(662) 31262 75, Email; isap_ac@hotmail.com
} 
de forma personal y comprendió preguntas de opción múltiple, aplicada a trabajadores de los ayuntamientos de la región de la sierra alta, seleccionando una muestra de 16 ayuntamientos de un universo de 17; el requisito para seleccionar al entrevistado consistía en que estuvieran relacionados con los presupuestos, es decir, que programaran, ejecutaran o supervisaran recursos del municipio al cual pertenece. La conjugación de todos estos elementos nos dará la pauta para determinar la eficiencia hacendaria que priva en el uso de los recursos públicos.

Palabras claves: Presupuesto, Eficiencia, trasparencia, PBR. BUDGET BASED ON RESULTS, (PBR) TOOL THAT CONTRIBUTES TO TRANSPARENCY AND EFFICIENCY IN MEXICO

\section{Abstract}

The purpose of this paper is to outline the current situation that prevails in the three levels of government, in relation to the use of the PBR, in addition to determining what has been the progress in the implementation of the Result Based Budgets, and in in its case, the budgetary amounts that have been objects of MIR at the dependencies or secretaries level. Regarding the mountain region of Sonora, it was sought to determine if there is knowledge and use of the PBR, which would mean that the municipalities are measuring the effectiveness in the application of public spending. The present work was carried out based on different techniques and strategies for data collection, in the case of the federal and state levels, documentary research was used. For local effect, the predominant technique was referred to as a survey, which was applied in a personal way, which included multiple choice questions, applied to workers of the municipalities of the high mountain region, selecting a sample of 16 municipalities of a universe of 17 , the requirement to select the interviewee consisted in that they were related to the budgets, that is to say that they programmed, executed or supervised resources of the municipality to which they belong. The conjugation of all 
these elements will give us the guideline to determine the tax efficiency that deprives in the use of public resources.

Key Words: Budget, Efficiency, Transparency, PBR

\section{Introducción/Revision de Literatura}

El objetivo del presente trabajo es determinar la situación actual del uso de la metodología del Presupuesto Basado en Resultados, (PBR) en los ámbitos Federal, Estatal y Local, para lo cual desarrolla un análisi histórico, hasta conocer el avance en la implantación del PBR.

Los procesos de modernización administrativa históricamente han estado evolucionando a nivel mundial. En México, el sistema de modernización administrativa se inició en 1965 al crearse la comisión de administración pública en el seno de la Secretaria de la Presidencia (Quirogas 2009,18) ${ }^{3}$. Estas trasformaciones fueron evolucionando como resultado de diferentes elementos coyunturales, el hecho de que en nuestro país existiera un sistema político mexicano caracterizado por un esquema de partido hegemónico durante aproximadamente 70 años (González y Velazco, 2014) ${ }^{4}$, impidió que este desarrollo fuera acelerado, sin embargo, no fue sino hasta la década de 1980 que se hicieron esfuerzos importantes para controlar el gasto público a raíz de la crisis de la deuda (Pardo, 2000) ${ }^{5}$.

El Gobierno mexicano, consiente y convencido de desarrollar metodologías que diluciden cada vez más y con mayor precisión la

${ }^{3}$ Quirogas Leos Gustavo, Organización y Métodos en la Administración Publica (México: Trillas, 2009), 18

${ }^{4}$ González Gómez Javier y Velasco Sánchez Ernesto, La evolución del presupuesto basado en resultados en México, 2004-2012: transferencia e implantación de una política pública, Revista del CLAD Reforma y Democracia,(Febrero 2014) 117

${ }^{5}$ Pardo María del Carmen.2000). La evaluación gubernamental: prioridad política de 1982 a 1988”, en Lecturas básicas de administración y políticas públicas, José Luis Méndez (comp.), México, El Colegio de México 
planeación de los recursos de los gobiernos en sus tres niveles, en el año 2006, inicia la adecuación a los PBR, incluso la Constitución Política de los Estados Unidos Mexicanos se reforma para impulsar la obligatoriedad de la evaluación del gasto público (González y Velazco 2014, 117) ${ }^{6}$, modificando el artículo 134 constitucional, y estableciendo que los tres órdenes de gobierno deberán administrar los recursos públicos bajo principios de eficiencia, eficacia, economía, transparencia y honradez; posteriormente se modificó la Ley Federal de Presupuesto y Responsabilidad Hacendaria, donde en los artículos 85 y 110 se establece que los recursos que reciban los estados y municipios (no aplica para ramo 28) serán evaluados con base en indicadores estratégicos y de gestión, por instancias técnicas independientes de las instituciones que ejerzan dichos recursos, observando los requisitos de información correspondientes, posteriormente al año siguiente (2007) se realizó una reforma fiscal Hacendaria, que trajo como consecuencia previa, las modificaciones al menos de 6 artículos de la Constitución Política de los Estados Unidos Mexicanos, CPEUM, cuya finalidad consistía en dar certeza jurídica al PBR. Los cambios en la CPEUM, establecen que el Gobierno Federal debe ser evaluado y dar complimiento a los nuevos requerimientos constitucionales. Posteriormente en el 2008, se realizó una modificación a la estructura programática con el propósito de alinear de forma más precisa los objetivos del Plan Nacional de Desarrollo y de los programas sectoriales con el sistema presupuestario (Bustamante 2013,50$)^{7}$, para lo cual se estableció que los objetivos e indicadores de los programas del gobierno mexicano (SAGARPA, SEDATU, SEDESOL, etc.), se enlazaran con el Plan Nacional de Desarrollo, (PND) y

6 González Gómez Javier y Velasco Sánchez Ernesto, La evolución del presupuesto basado en resultados en México, 2004-2012: transferencia e implantación de una política pública, Revista del CLAD Reforma y Democracia,(Febrero 2014) 124

${ }^{7}$ Bustamante Terreros Cesar. 2013 Ejercicio del Gasto Público; Experiencias Internacionales, Centro de Estudios de las Finanzas Públicas (Septiembre) 25 
este último documento lograr ser un real instrumento de verificación, donde se midieran los objetivos establecidos por las dependencias y su incidencia en la estrategia nacional del Plan Nacional de Desarrollo. Además esta verificación tendría que ser realizada por entes externos a las dependencias, y es bajo esa premisa que se creó el Sistema de Evaluación del Desarrollo (SED), el cual, se puede considerar la parte medular del PBR, en su definición el SED, es la relación de elementos metodológicos que permiten realizar una valoración objetiva y real del desempeño de los programas, mediante los cuales se analiza y comprueba el grado de cumplimiento de metas y objetivos, con base en indicadores, entonces un SED no está expresando listado de deseos o intenciones: está explicando a la sociedad los argumentos y supuestos, así como comprometiendo políticamente acciones y efectos.(Arellano, Lepore, Zamudio y Blanco 2014, 28) ${ }^{8}$. Esto trae como consecuencia que gracias al SED, se logre un seguimiento puntual del avance en los objetivos de cada dependencia, asegurando que el ejercicio de los recursos ocurra de manera efectiva para lograr los objetivos establecidos en la etapa de planeación. La experiencia mexicana en la adopción del PBR ha concedido enorme importancia a la parte de la evaluación y la rendición de cuentas, incluida la trasparencia como prerequisito. (Acevedo, 2014) ${ }^{9}$ Es decir, contar con un Presupuesto Basado en Resultados permite que los programas sean evaluados por la Comisión Nacional de Evaluación de la Política Social y por la misma Secretaria de Hacienda, a través del SED, en los ámbitos legales que corresponden a cada una de ellas.

8 Arellano Gault David, Walter Lepore Emilio Zamudio y Blanco Felipe, Sistema de Evaluación del Desempeño para organizaciones públicas: ¿Cómo construirlas efectivamente? (México: Centro de Investigaciones en Docencia Economica, 20xx), 18

9 Arellano Gault David, Walter Lepore Emilio Zamudio y Blanco Felipe, Sistema de Evaluación del Desempeño para organizaciones públicas: ¿Cómo construirlas efectivamente? (México: Centro de Investigaciones en Docencia Económica, 20xx), 18 
Derivado del análisis de todos los elementos anteriores, la finalidad del presente trabajo es responder a las preguntas de, ¿Cuál es el avance en la implementación del PBR en los ámbitos federal, estatal y específicamente en el local?, además determinar los montos en donde actualmente se aplica el PBR y por ultimo analizar la disposición que existe por los distintos actores locales a usar el PBR en los presupuesto asignados.

\section{Materiales Y Métodos}

\section{Determinación del tipo de estudio}

El presente trabajo consta de dos tipos de estudio, el primero es para el caso del avance de aplicación o uso de la metodología PBR, en los presupuestos a nivel Federal y Estatal se realizó una investigación documental, para determinar la obligación legal y segundo, determinar el avance en la implementación de los presupuesto a nivel dependencia.

A nivel local, dada la poca disponibilidad de la información, se determinó un estudio descriptivo, lo que nos ayudó conocer los factores que originan que los ayuntamientos de la sierra alta de Sonora implementen o no, los Presupuestos Basados en Resultados.

\section{Estrategias y técnicas de recolección de datos a nivel local}

Como estrategia se realizó una encuesta a trabajadores de los $\mathrm{H}$. Ayuntamientos, donde el requisito indispensable consistía en que el trabajador trabajara de manera directa en la elaboración, aprobación o ejecución de los presupuestos operados por el H. Ayuntamiento en cuestión. Para recabar información fue la aplicación de una encuesta a una muestra de 16, de una población total que comprende 17 Ayuntamientos de la sierra alta. La cual fue aplicada de manera directa y de forma personal. Este instrumento contiene 11 preguntas de opción múltiple, la cual fue aplicada a los empleados de los distintos municipios seleccionadas, a su vez el método 
que se utilizo fue el método no probabilístico y con referencia de una base de datos que tiene la entidad de los apoyos que se entregaron los últimos tres años.

La fuente primaria utilizada fue la encuesta realizada a los empleados de los H. Ayuntamiento, y la Secundaria la investigación información obtenida de la investigación documental. Las cuales nos proporcionaron las bases para detectar aspectos relevantes del uso de Presupuesto Basado en Resultados.

A continuación, se explica el proceso para determinación de la muestra para determinar el número de personas a las que se les aplicó la encuesta:

$$
\begin{gathered}
n=\text { p.q. } N \\
\mathrm { e } ^ { 2 } ( \mathrm { N } - 1 ) \longdiv { + \text { p. q. } \mathrm { S } ^ { 2 } }
\end{gathered}
$$

$S=$ Nivel de confianza $=95 \%=1.96$

$\mathbf{p}=$ Variabilidad positiva $=0.5$

$\mathbf{q}=$ Variabilidad negativa $=0.5$

$\mathbf{N}=$ Población $=17$

$e=$ Margen de error $=5 \%=0.05$

$\mathrm{n}=$

$$
(0.05)^{2}(17-1)+(0.5)(0.5)(1.96)^{2}
$$

$\mathrm{n}=$

$(3.8416)(0.5)(0.5)(985)$

$(0.0025)(16)+(0.5)(0.5)(3.8416)$

$\mathrm{n}=\frac{16.32}{1.0004}=16$ encuestas 
Para determinar los factores que influyen en el uso de PBR por los actores encargados de su implementación, fue necesario la representación gráfica de la información, para una mayor comprensión de los resultados, mismos que se derivan de la aplicación de las 16 encuestas.

\section{Marco normativo y operacional del PBR y las Matrices de Indicadores de Resultados (MIR), en el ámbito federal.}

EI SED para estar en posibilidades de realizar una correcta evaluación, solicita a los entes obligados el establecer una Matriz de Indicadores de Resultados (MIR), la cual es un instrumento para el diseño, organización, ejecución, seguimiento, evaluación y mejora de los programas, la Unidad de Desempeño, Dependiente de la Subsecretaria de Egresos, de la SHCP, es quien de manera anual y a través del oficio 419-A-17-0609 de fecha 30 de junio del 2017, determinó los criterios que deberán contener las actualizaciones y registros de las MIR de los programas presupuestales de 2018, que lleven a cabo las dependencias del gobierno federal y las entidades de los estados, también establece que todos los programas presupuestales a ejercer en el 2018, deberán de contar con una MIR registrada en el modelo PBR (salvo casos de programas presupuestales de desarrollo social, donde CONEVAL define la procedencia de la exención).(SHCP) $)^{10}$

Las MIR son registradas en un Sistema de Presupuesto Basado en Resultados y Evaluación del Desempeño, el cual, es administrado por la

\footnotetext{
${ }^{10}$ SHCP. Ver Secretaría de Hacienda y Crédito Público. CÉDULA: conceptos básicos del presupuesto basado en resultados- sistema de evaluación del desempeño (PBR-SED). http://www.shcp.gob.mx/EGRESOS/sitio_pbr/Documents/090811CeduladelP bR-SED.pdf.
}

(Consultada el 01 de Enero del 2018). 
Secretaria de Hacienda, y se puede acceder al mismo desde un explorador de internet con la dirección del sitio http://www.sistemas.hacienda.gob.mx/PASH/jsps/acceso.jsp.(SHCP).

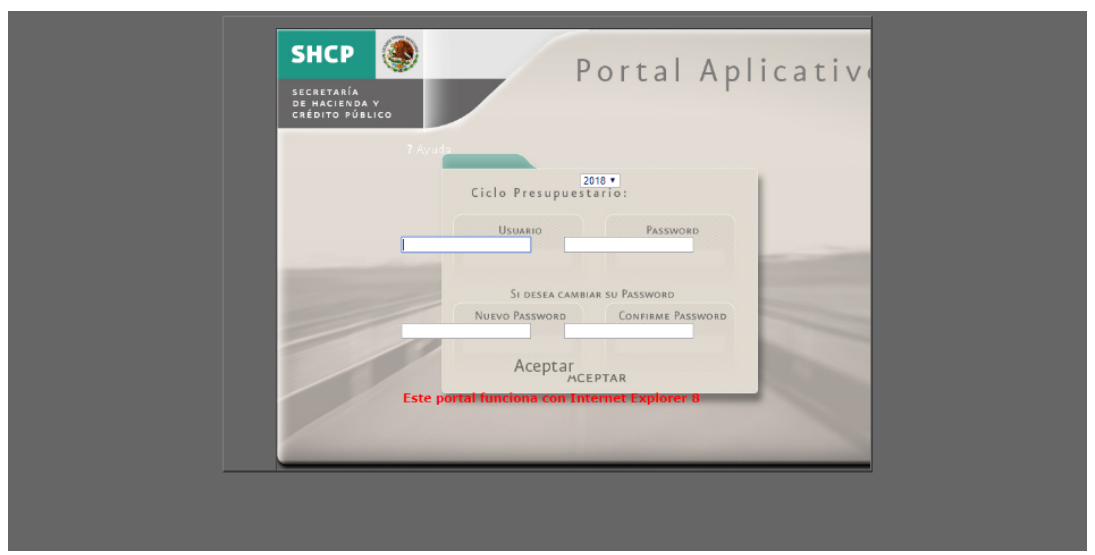

Este portal donde se registran las MIR, al acceder y para poder crear una matriz, usa como base la Metodología de Marco Lógico (MML), la cual, nos permite alinear la programación de la dependencia o sector con los objetivos del Plan Nacional de Desarrollo, y de esta manera, verificar el alcance logrado. Esto lo hace a través de una serie de pasos mediante los cuales se van llenando los campos que establece el sistema, (Guía de Operación Sistema de Evaluación del Desempeño), y nos va dando la pauta para llevar de forma sistemática y acorde a los requerimientos normativos, el proceso de llenado de las MIR, posteriormente nos permitirá, realizar una valoración objetiva del desempeño de los programas presupuestarios, mediante la verificación del grado de cumplimiento de metas y objetivos, teniendo como elemento primordial, la serie de Indicadores del Desempeño estructurados en Matrices y, que previamente fueron capturados en el portal del SED.

\section{Marco Normativo y operacional del PBR y las MIR a nivel Estatal}

Los estados y municipios están obligador por ley a realizar la presupuestación basada en resultados, conforme lo establece el artículo 134 
de la Constitución Política de los Estados Unidos Mexicanos. Pero además de esta reglamentación, existe el artículo 85 de la Ley Federal de Presupuestación y Responsabilidad Hacendaria, LFPRH., entre lo que destaca:

- Establece que los recursos federales que ejerzan las entidades federativas y los municipios, se evaluará con base en indicadores estratégicos y de gestión, por instancias técnicas independientes de las instituciones que ejerzan dichos recursos.

Dado lo anterior, los estados se han comprometido con el SED, (además es obligación jurídica) a evaluar el cumplimiento estratégico de sus objetivos mediante indicadores y metas. Lo anterior ha permeado en diferentes magnitudes en los estados de la república mexicana.

Para el caso de Sonora y con la finalidad de dar cumplimiento en este rubro, se determina en primera instancia que se realice la alineación de los ejes estratégicos del gobierno federal (2013-2018), con el plan Estatal de Desarrollo (2016-2021). (Secretaria de Economía) ${ }^{11}$

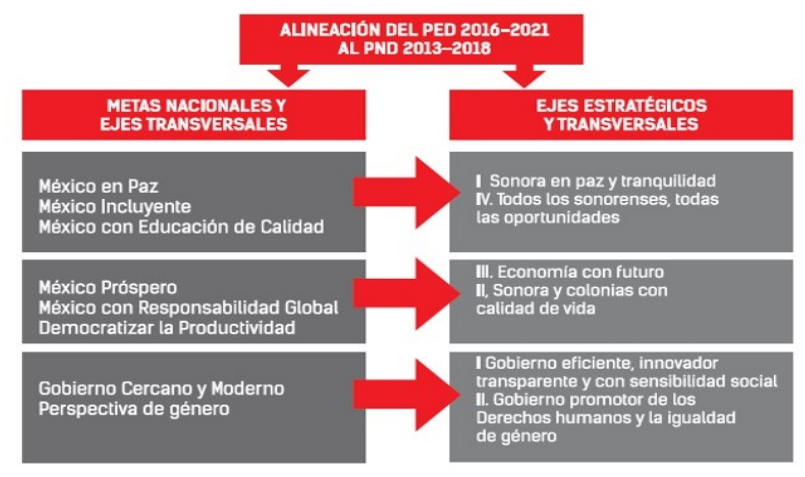

(Fuente: Programa Estatal de Desarrollo 2016-2018)

${ }^{11}$ SE. Ver Secretaría de Economía. Plan Estatal de Desarrollo 2016-2021: Sonora trabaja.

https://prosoft.economia.gob.mx/organismos/docop/ESTRATEGIAS\%20OP \%20SONORA\%202016.pdf (consultada el 10 de enero del 2018). 
Posteriormente en el presupuesto de egresos del estado de Sonora del 2017, se estableció que las etapas de ciclo presupuestal, serian alineadas de manera gradual conforme al modelo federal a efecto de poder homologar el PBR-SED, y posteriormente se emitieron lineamientos para las dependencias estatales, a efecto de que en este contexto las mismas, emitieran las matrices de indicadores de resultados.

\section{Resultados y Discusión}

En el caso del presupuesto de egresos del Estado de Sonora, el presupuesto del ejercicio 2018, tiene un importe autorizado por el Congreso de 64 mil 17 millones 94 mil pesos, del monto total podemos ver que $\$ 34,578,094,000.00$ agrupa las dependencias que tienen MIR, lo que nos da un porcentaje del $52 \%$ del total de presupuesto con MIR, al efecto habría que añadir, que los recursos por participaciones federales no son objeto de obligatoriedad para elaboración de MIR.

Al realizar el análisis por dependencia para este ejercicio fiscal se elaboraron un total de $40 \mathrm{MIR}$, agrupadas en 9 dependencias del gobierno del estado, siendo la Secretaria de Educación y Cultura, la dependencia estatal que agrupa más MIR, en el estado de Sonora (cuadro 1), y el Tribunal de Justicia Administrativo, el organismo que tiene menos MIR, siendo el total de 1. (Congreso Sonora) ${ }^{12}$

\footnotetext{
${ }^{12}$ Ver_Congreso del Estado de Sonora. Presupuesto de Egresos; Matrices de Indicadores de Resultados 2018.

http://www.congresoson.gob.mx/Content/PE2018/pdfs/matrices.pdf (consultada el 01 de enero del 2018).
} 


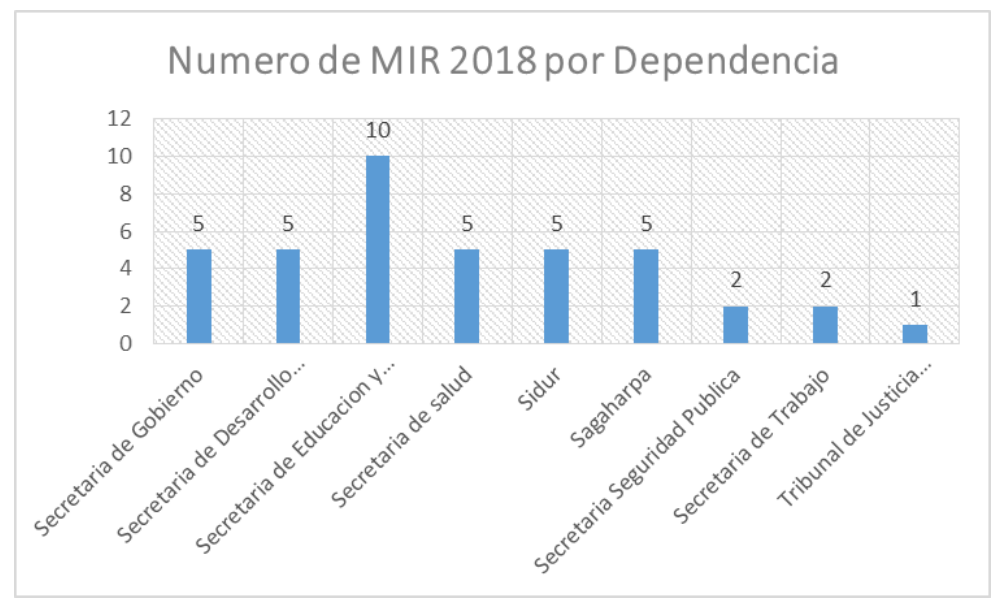

Grafico 1 (Elaboración propia)

Al realizar el comparativo en base a montos presupuestales asignado por dependencia, tenemos que la media, en relación al total presupuestal con MIR, corresponde a un monto de 864,474 millones por MIR, en este sentido la dependencia que tiene mayor número de MIR, en base a los montos presupuestales medios, corresponde al Tribunal de Justicia Administrativa y en segundo lugar se encuentra la Secretaria del Trabajo con montos de 68,827.00 y 70,595.50 millones, en relación contraria, podemos ver que la Secretaria de Educación y Cultura, aun cuando tiene mayor número de MIR, con 10 en total, la relación MIR-presupuesto, ubica al doble de la media presupuestal de presupuesto por cada MIR medio del total con importes de 1,199,910.8 


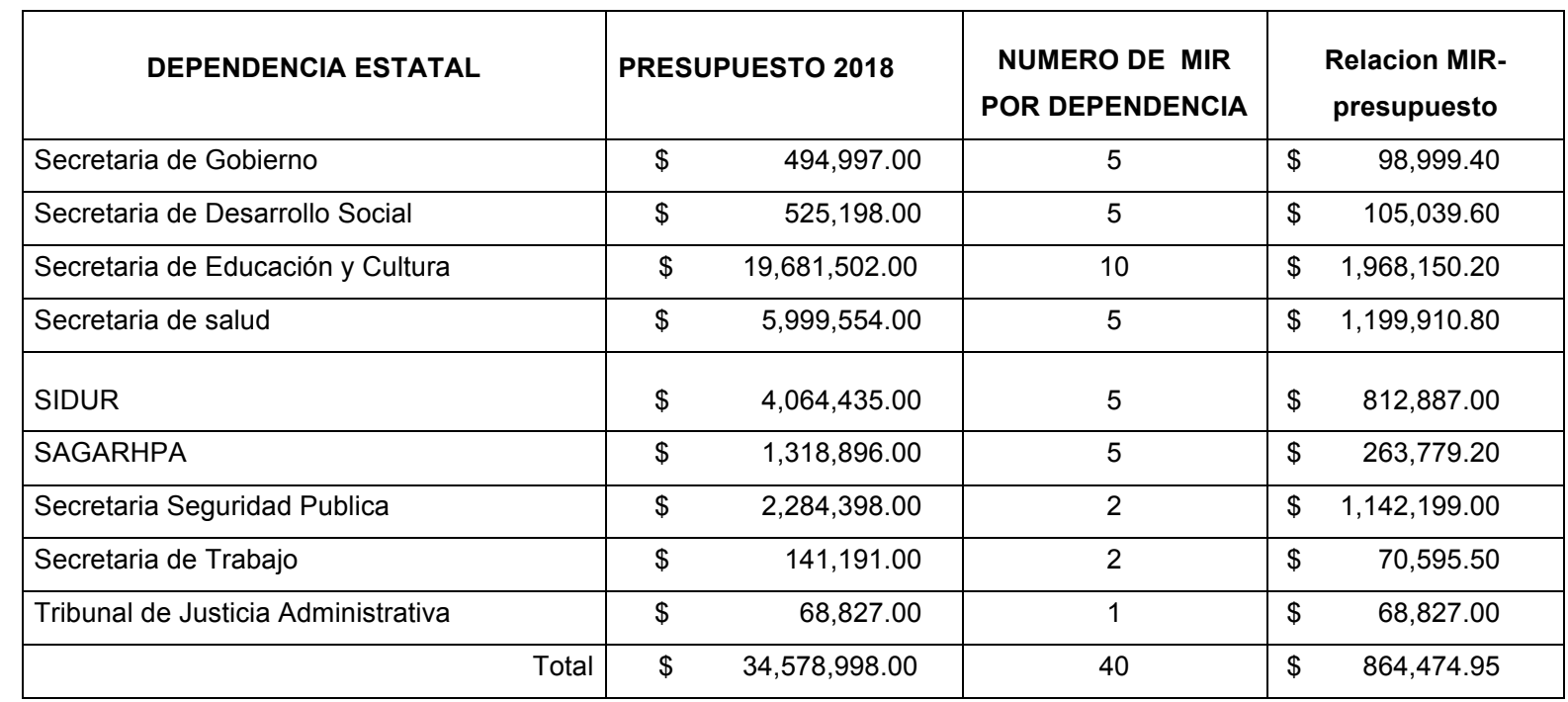

\section{Cuadro 1 (elaboración propia)}

Esto se debe a que las MIR, se elaboraron por programas presupuestales que tienen agrupadas las dependencias del Gobierno del Estado, sin embargo, es un referente importante para denotar la importancia de las MIR a nivel dependencia, y hace énfasis en la revisión de los indicadores de aquellas MIR que por los montos presupuestales sea más cuantiosas, además de otros elementos inherentes al programa en sí.

Al realizar un esbozo final de la situación del PBR-SED para el estado de Sonora, podemos ver que a partir del 2017, esta metodología fue impulsada por más voluntad de los actores institucionales que forman parte del proceso, así lo demuestran la creación de las normatividades, los sistemas de seguimiento, la información disponible para los ciudadanos, y las MIR disponibles, la otra parte que aún falta es darle seguimiento a las MIR, a efecto de evaluar el cumplimiento de los indicadores y metas propuestos por las dependencias, lo cual se considera será la siguiente hoja del PBR-SED. 


\section{Situación actual del PBR y las MIR en la región Sierra Alta de}

\section{Sonora.}

Los resultados obtenidos de los cuestionarios aplicados a los municipios de la sierra, se desglosa a continuación.

La primer pregunta, referida a su participación en la elaboración del presupuesto basado en PBR, todos los entrevistados respondieron que sí. Otra de las preguntas que se consideró importante, era en referencia al conocimiento que tenían del PBR-SED, y el $86 \%$ de los entrevistados mencionó conocer el SED y el funcionamiento del PBR.

En relación a la pregunta de, ¿en el municipio utilizan la presupuestación basada en resultados?, en $71 \%$ de los municipios menciono que si la utilizaban y $29 \%$ no utilizaban el PBR, tal como se refleja en el gráfico 3.

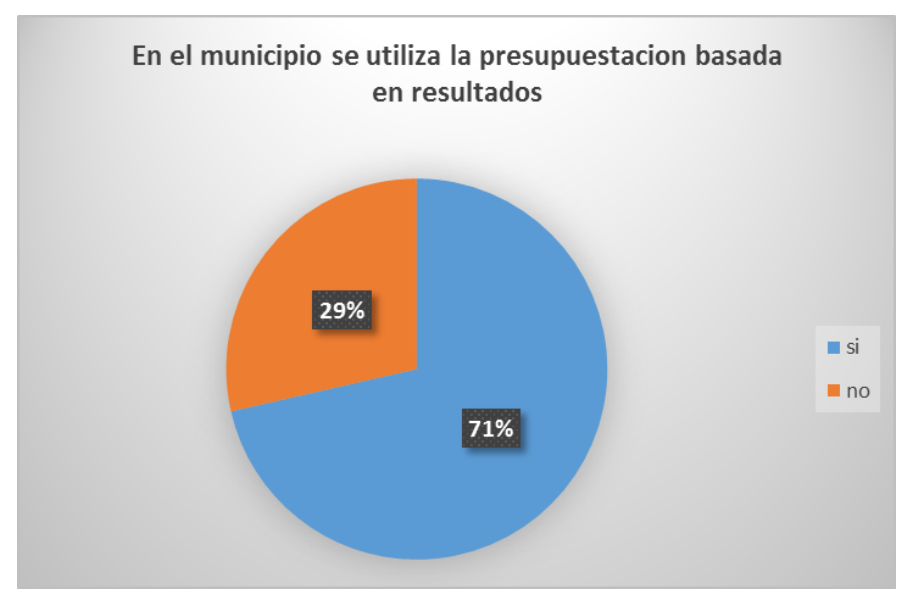

Grafico 3, (elaboración propia)

Otra de las preguntas, que se realizó a los entrevistados, fue sobre el uso de las MIR, durante el proceso presupuestal inherente a cada municipio. En relación a este punto el $41 \%$ de los entrevistados mencionaron, que efectivamente en su municipio la utiliza, lo que corresponde a 7 municipios del total de los 16 (grafico 4), en esta pregunta, fueron más los municipios, que a pesar de conocer los el 
PBR-SED, no utilizan las matrices para poder establecer indicadores y metas a efecto de ser más eficiente en el ejercicio de los recursos.

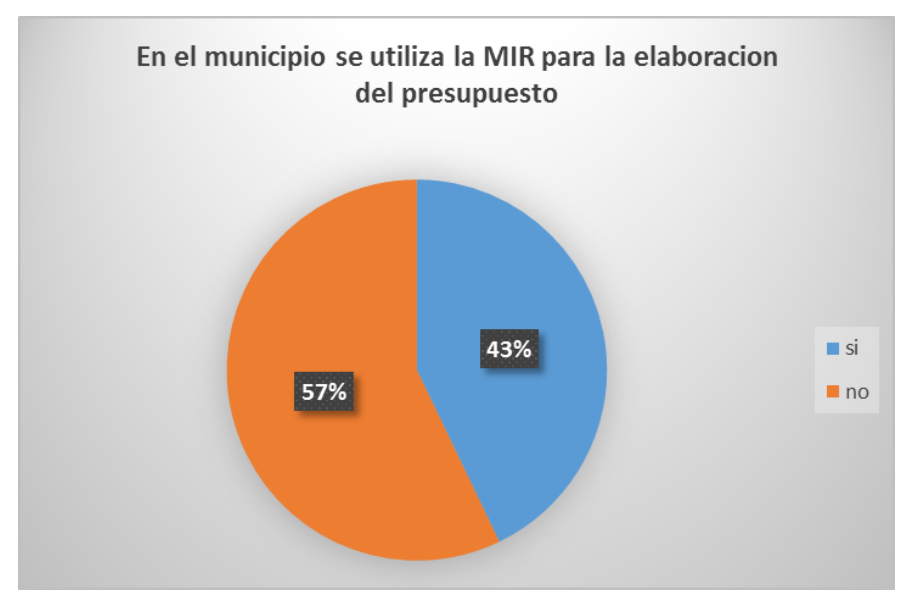

Grafico 4 (elaboración propia)

A los encargados de presupuestar, también se les preguntó que si les parecía bien o mal el PBR, el 100\% de entrevistados mencionó, que les parecía bien y que si utilizan la metodología del Presupuesto Basado en Resultados.

A los mismos entrevistados se les realizó la última pregunta, cuya intención era conocer el grado de calificación que le daban al PBR, desde un enfoque administrativo y de operación cotidiana, además de asignarle una calificación cuantitativa del 1 al 10 , el $37 \%$ le otorgaba un 10 , el $38 \%$ le asignaron un 9 y el $25 \%$ un 8 , es decir que todos los funcionarios públicos municipales de la región serrana que hacen uso de los presupuestos en alguna de sus respectivos municipios, consideran que el PBR, es bueno, ya que mencionaron rangos máximos $(8,9$ y 10) tal como se aprecia en la gráfica número 5 . 


\section{Conclusiones}

Ha trascurrido más de una década desde que se dieron los primeros indicios de los Presupuestos Basados en Resultados, esta modalidad, se ha ido adecuando a los contextos de nuestra cultura gubernamental, y poco a poco ha ganado terreno, lo vemos desde el ámbito jurídico, incluyendo las modificaciones constitucionales, y posteriormente en las leyes secundarias y sus reglamentos, además de la serie de oficios, memorándums y documentos tales como manuales de presupuestos, también de informes, presupuestos de egresos de los congresos locales y en el académico en el crecimiento y desarrollo de investigaciones, ensayos y cursos de capacitación.

Los montos presupuestales que operan con PBR se han incrementado en los ámbitos federales y estatales, podemos ver como permean en los informes de las secretarias, y de los gobiernos federales, transformándose en una cultura más perene. En el caso de nuestro análisis a nivel local, desarrollado en la región serrana de Sonora, podemos ver que existe conocimiento por parte de la mayoría de las autoridades encargadas de operar los presupuestos municipales, reflejando cierta resistencia por los sujetos obligados a su aplicación, esto se debe a factores como falta de conocimiento en su instrumentación, o desconocimiento de las leyes, sin embargo, y como resultado de la investigación, es un hecho concreto, que el PBR está operando en la región serrana, solo es cuestión de sensibilización y que se den los tiempos para lograr su implementación eficiente y una vez aplicado, poco a poco identificaremos la eficacia y trasparencia de recursos y presupuestos públicos, causando por ende, la mejora en el bienestar de la población serrana de Sonora.

\section{Referencias Bibliográficas}

Acevedo Márquez Daniel Arturo, Ejercicio del Gasto Público: El Presupuesto Basado en Resultados, Caso México, Centro de Estudios de las Finanzas Públicas, Septiembre,2013 
Arellano Gault David, Walter Lepore Emilio Zamudio y Blanco Felipe, Sistema de Evaluación del Desempeño para organizaciones públicas: ¿Cómo construirlas efectivamente? México, Centro de Investigaciones en Docencia Económica 2012

Bustamante Terreros Cesar, Ejercicio del Gasto Público: Experiencias Internacionales, Centro de Estudios de las Finanzas Públicas Septiembre,2013

CONGRESOSON. Ver Congreso del Estado de Sonora, Presupuesto de Egresos; Matrices de Indicadores de Resultados 2018 [en línea] Disponible en:

http://www.congresoson.gob.mx/Content/PE2018/pdfs/matrices.pdf (consultada el 01 de enero del 2018).

HACIENDASONORA. Ver_Secretaría de Hacienda Sonora. Proyecto de Presupuesto de Egresos 2018; Exposición de Motivos e Iniciativa de Decreto. Gobierno del Estado de Sonor, 2018 [en línea] Disponible en:

http://hacienda.sonora.gob.mx/media/2577/exposicion.pdf_(consultada el 08 de enero del 2018).

González Gómez Javier y Velasco Sánchez Ernesto, La evolución del presupuesto basado en resultados en México, 2004-2012: Transferencia e implantación de una política pública. Revista del CLAD Reforma y Democracia, (Febrero) 2014

Quirogas Leos Gustavo, Organización y Métodos en la Administración Pública. México: Trillas,2009

SE. Ver Secretaría de Economía. Plan Estatal de Desarrollo 2016-2021: Sonora trabaja,2016[en línea] Disponible en:

https://prosoft.economia.gob.mx/organismos/docop/ESTRATEGIAS\%200P \%20SONORA\%202016.pdf (consultada el 10 de enero del 2018).

SHCP. Ver Secretaría de Hacienda y Crédito Público. CÉDULA: conceptos básicos del presupuesto basado en resultados- sistema de evaluación del desempeño PBR-SED [en línea] Disponible en:

http://www.shcp.gob.mx/EGRESOS/sitio_pbr/Documents/090811CeduladelP bR-SED.pdf_(consultada el 01 de Enero del 2018).

Pardo María del Carmen. La evaluación gubernamental: prioridad política de 1982 a 1988", en Lecturas básicas de administración y políticas públicas, José Luis Méndez (comp.), México, El Colegio de México, 2000

Recibido 17 mayo 2018 Aceptado 18 Octubre 2018 